\title{
Wild Birds as Hosts of Amblyomma cajennense (Fabricius, 1787) (Acari: Ixodidae)
}

\author{
Rosario Rojas/ ${ }^{+}$, Miguel Ângelo Marini*, Maria Teresa Zanatta Coutinho**
}

Curso de Pós-graduação em Ecologia, Conservação e Manejo de Vida Silvestre *Departamento de Biologia Geral **Departamento de Parasitologia, Instituto de Ciências Biológicas, Universidade Federal de Minas Gerais,

Av. Antônio Carlos 6627, 30161-970 Belo Horizonte, MG, Brasil

We evaluated the prevalence, mean intensity and relative density of ticks in 467 wild birds of 67 species (12 families) from forest and cerrado habitats at two protected areas of Minas Gerais, between March and September 1997. Ticks collected $(n=177)$ were identified as larvae and nymphs of Amblyomma cajennense and four other species of Amblyomma. We report for the first time 28 bird species as hosts of the immature stages of A. cajennense, demonstrating the lack of host specificity of the larvae and nymphs. A. cajennense had $15 \%$ prevalence on birds, with a mean infestation intensity of 0.37 ticks per host sampled, and 2.5 ticks per infested bird. Prevalence varied in relation to host species, diet and between birds from forests at two successional stages. There were no differences in relation to host forest dependence, participation in mixed flocks of birds, and nest type constructed. A. cajennense is a species of medical and veterinary importance, occurring on domestic animals but is known little of its occurrence on wildlife.

Key words: Amblyomma cajennense - birds - wild hosts - larvae - nymphs

Amblyomma cajennense (Fabricius, 1787) is an ixodid tick which parasitizes man, domestic and wild animals and is very common in Brazil. It occurs in the New World from southern South America to southern North America (Hoogstraal \& Aeschlimann 1982) and is a known vector of Rickettsia rickettsii which causes Rocky mountain spotted fever in humans as well as being a possible vector of brucella and Trypanosoma cruzi in humans (Smith 1974). It is also a possible vector of piroplasmosis in cattle and has been demonstrated to cause hematological alterations in parasitized cattle (Serra Freire \& Cunha 1987).

The life cycle of $A$. cajennense has four stages: egg, hexapod larvae, octopod nymph and adult (Walker 1994). The cycle of A. cajennense during its free stages and as a parasite of domestic species has been studied primarily under controlled laboratory conditions by Rohr (1909), Olivieri and Serra Freire (1984a, b), Souza and Serra Freire (1992), Serra Freire and Olivieri (1992), and Lopes et al. (1998)

This investigation was supported by Fapemig, PRPq and CNPq.

${ }^{+}$Corresponding author. Fax: +55-31-499.2567. E-mail: rosario@dedalus.lcc.ufmg.br.

Received 24 July 1998

Accepted 1 February 1999
A. cajennense is considered an eclectic ectoparasite, both during its immature stages and possibly during its adult stages, with a wide range of hosts (Hoogstraal \& Aeschlimann 1982). Adults usually parasitize equids, cattle and dogs as well as several other domestic species. This species has also been found on several wild vertebrates (Aragão 1936), including rodents (Linardi et al. 1987), edentates (Botelho et al. 1989) and several other species of small mammals (Coutinho 1997).

There is lack of studies on the role of wild birds on the dispersal of immature stages of $A$. cajennense. This study presents data on wild birds as hosts and dispersers of immature stages of $A$. cajennense. It evaluates the prevalence, mean intensity and relative density of infestation on birds from forests and cerrado habitats of Minas Gerais, correlating the infestation indexes with host taxonomy, environmental variables and ecological characteristics of the birds.

\section{MATERIALS AND METHODS}

This study was conducted at "Áreas de Proteção Especial (APE) para fins de Preservação de Mananciais" owned by "Companhia de Saneamento de Minas Gerais" (Copasa), $\left(43^{\circ} .50^{\prime} \mathrm{W}, 19^{\circ} .50^{\prime} \mathrm{S}\right)$. Two study areas were sampled (1) Mutuca APE (1,250 ha) and (2) Barreiro APE (1,406 ha), located in the municipalities of Nova Lima and Belo Horizonte, respectively. Vegetation at these areas is composed of cerrado, gallery forest, and dry forest (Cetec 1996). 
At Barreiro APE, the forest is a more developed dry tropical forest at a more advanced successional stage ( $150 \mathrm{yrs})$ than the Mutuca APE forest which has gallery and dry forest at an earlier successional stage ( 90 yrs) (Cetec 1996).

The region has distinct seasonal weather changes with warm rainy summers between November and March and cool dry winters between April and October (Cetec 1983) (Fig. 1). During 1997, the study sites had a total precipitation of $2,244 \mathrm{~mm}$. The mean air temperature varied seasonally between $18^{\circ} \mathrm{C}$ and $24^{\circ} \mathrm{C}$, July being the coolest month with mean air temperature between $14^{\circ} \mathrm{C}$ and $22^{\circ} \mathrm{C}$ (Sistema de Controle Climatológico Copasa).

Birds were captured at forest fragments of 1,2 , 32, and 150 ha at Barreiro and at a 200 ha fragment at Mutuca. Areas sampled included forest interior, the edge of these forests and cerrado transects in Mutuca and Barreiro. Captures were conducted between March and September 1997, with $15-17$ mist nets $(36 \mathrm{~mm}$ mesh, and $12 \mathrm{~m}$ long by $2,5 \mathrm{~m}$ high), placed close to the ground and opened between 7:00 and 16:00 three to four days a week, in a different area each week. Birds were identified with the help of field guides (Hilty \& Brown 1986, Dunning 1987, Ridgely \& Tudor 1989 a, b). Each bird captured was weighed, metal and color banded, measured (tail, wing, tarsus, and culmen), checked for the presence of molt, brood patch, sexed when possible, and aged through cranial ossification. Ticks were sampled checking mostly the head, neck, and belly, because these are the areas were ticks are most often found attached to birds. Ticks were collected from the body of birds with forceps and preserved in $70 \%$ alcohol. Birds were released after sampling. For the identification of ticks we used the Clifford and Anastos (1960) and Famadas et al. (1997).

Each bird species had its prevalence (number of individuals of a host species infected with a parasite species divided by the number of hosts examined), and its mean intensity (mean number of individuals of a parasite species per infected host in a sample) and relative density (mean number of individuals of a parasite species per host examined) (Margolis et al. 1982). Prevalence was compared with respect to bird taxon, diet, participation in mixed species flocks (feeding aggregations of birds), nest type constructed, and sex. Environmental variables such as level of forest dependence, forest edge effects, forest size effects, and seasonal variation, were also tested for differences in tick prevalence. The prevalence of ticks on birds and the variables considered were tested with $\mathrm{Chi}$ square tests using Yates correction $\left(\mathrm{C}_{Y}{ }^{2}\right)$ when degrees of freedom were 1. Spearman rank correla- tion $\left(r_{s}\right)$ was used to compare prevalence and forest size and prevalence and medium infestation intensity. Statistical analyses were considered significant at $5 \%$.

\section{RESULTS}

Tick prevalence, mean intensity and relative density were evaluated for 436 birds of 59 species (9 families) of Passeriformes and 31 bird species (3 families) of non-Passeriformes (Tables I, II). Ticks collected $(n=177)$ were identified as larvae and nymphs of $A$. cajennense and also of four other unidentified species of Amblyomma. Adults were never found on birds.

A. cajennense was present on 28 bird species (Table I), and four other unidentified Amblyomma species were found on the following birds: Turdus albicollis, Pyriglena leucoptera, Tolmomyias sulphurescens, Basileuterus flaveolus, Conopophaga lineata and Saltator similis.

Passeriformes and non-Passeriformes showed differences in prevalence values, since no ticks were found on the 31 non-passerine birds sampled whereas the passerines had a prevalence of $16 \%$ (Table II). Prevalence values varied among bird families, being highest on Formicariidae (25\%) and Pipridae (20.9\%). The values of the mean infestation intensity were high for all passeriform families, being highest on Pipridae (5.1), Vireonidae (5.0) and Dendrocolaptidae (2.66). The highest relative infestation intensity was detected on Pipridae (1.07), Vireonidae (0.83) and Formicariidae (0.53) (Table I).

Tick prevalence varied significantly $\left(c^{2}=64.573 ; \mathrm{gl}=4 ; \mathrm{p}=0.000\right)$ among bird dietary groups being highest on omnivorous $(21.2 \%)$ and lowest on nectarivorous ( $0 \%$ ) birds. Birds that participate in mixed-species flocks did not differ in tick prevalence $\left(\mathrm{C}_{\mathrm{Y}}{ }^{2}=0.16 ; \mathrm{df}=1 ; \mathrm{p}=0.691\right)$ from those that do not participate. Also, birds nest type did not show any significant relationship $\left(c^{2}=0.449 ; \mathrm{df}=2 ; \mathrm{p}=0.975\right)$ with $A$. cajennense prevalence. Tick prevalence also did not vary in relation to host sex. This pattern was evident both for the species with more than 10 individuals sampled as well as for males and females of all species pooled together $\left(\mathrm{c}_{Y}{ }^{2}=0.38 ; \mathrm{df}=1, \mathrm{p}=0.539\right)$. Birds with different levels of forest dependence did not show different prevalence of $A$. cajennense $\left(c^{2}=2.73 ; \mathrm{df}=2 ; \mathrm{p}=0.200\right)$.

No significant difference was detected in tick prevalence between birds captured at the interior and at the edge of the forests (Table III). Tick prevalence also did not vary significantly between birds captured at forests and at cerrado formations (Table III). The correlation between tick prevalence and forest size was also not significant $\left(r_{s}=0.60\right.$; 
TABLE I

Prevalence, mean and relative infestation intensity of Amblyomma cajennense, on forest and cerrado birds at Barreiro and Mutuca, Minas Gerais, between March and September 1997

\begin{tabular}{|c|c|c|c|c|c|c|c|c|c|c|c|c|c|}
\hline $\begin{array}{l}\text { Family } \\
\text { (species) }\end{array}$ & $\begin{array}{l}\text { Individuals } \\
\text { examined }\end{array}$ & Prevalence $^{a}$ & $\begin{array}{l}\text { Number } \\
\text { of ticks }\end{array}$ & $\begin{array}{l}\text { Relative } \\
\text { density }\end{array}$ & $\begin{array}{c}\text { Mean } \\
\text { intensity }\end{array}$ & Variation $^{d}$ & Diet $^{e}$ & Nest $^{f}$ & $\begin{array}{l}\text { Mixed } \\
\text { species } \\
\text { flocks } g\end{array}$ & $\begin{array}{c}\text { Forest } \\
\text { dependence }^{h}\end{array}$ & $\begin{array}{l}\text { Forest } \\
\text { interior }\end{array}$ & $\begin{array}{l}\text { Forest } \\
\text { border }\end{array}$ & Cerrado \\
\hline $\begin{array}{l}\text { Non Passeriformes } \\
\text { Columbidae (1) } \\
\text { Leptotila rufaxilla }\end{array}$ & $\begin{array}{l}2 \\
2\end{array}$ & $\begin{array}{l}0(0) \\
0(0)\end{array}$ & $\begin{array}{l}0 \\
0\end{array}$ & $\begin{array}{l}0 \\
0\end{array}$ & - & $\begin{array}{l}0 \\
0\end{array}$ & $\mathrm{~F}$ & A & $\mathrm{N}$ & 2 & $X$ & $X$ & \\
\hline $\begin{array}{l}\text { Trochilidae (6) } \\
\text { Phaetornis pretrei } \\
\text { Colibri serrirostris } \\
\text { Chlorostilbon aureoventris } \\
\text { Thalurania furcata } \\
\text { Amazilia } \text { sp. } \\
\text { Amazilia lactea }\end{array}$ & $\begin{array}{r}28 \\
2 \\
1 \\
1 \\
18 \\
5 \\
1\end{array}$ & $\begin{array}{l}0(0) \\
0(0) \\
0(0) \\
0(0) \\
0(0) \\
0(0) \\
0(0)\end{array}$ & $\begin{array}{l}0 \\
0 \\
0 \\
0 \\
0 \\
0 \\
0\end{array}$ & $\begin{array}{l}0 \\
0 \\
0 \\
0 \\
0 \\
0 \\
0\end{array}$ & $\begin{array}{l}- \\
- \\
- \\
-\end{array}$ & $\begin{array}{l}0 \\
0 \\
0 \\
0 \\
0 \\
0 \\
0\end{array}$ & $\begin{array}{l}\mathrm{N} \\
\mathrm{N} \\
\mathrm{N} \\
\mathrm{N} \\
\mathrm{N} \\
\mathrm{N}\end{array}$ & $\begin{array}{l}\text { A } \\
\text { A } \\
\text { A } \\
\text { A } \\
\text { A } \\
\text { A }\end{array}$ & $\begin{array}{l}\mathrm{N} \\
\mathrm{N} \\
\mathrm{N} \\
\mathrm{N} \\
\mathrm{N} \\
\mathrm{N}\end{array}$ & $\begin{array}{l}2 \\
2 \\
2 \\
2 \\
3 \\
3\end{array}$ & $\begin{array}{l}X \\
X\end{array}$ & $\begin{array}{l}X \\
X \\
X\end{array}$ & $\begin{array}{l}X \\
X \\
X \\
X \\
X\end{array}$ \\
\hline $\begin{array}{l}\text { Family Picidae (1) } \\
\text { Picumnus cirratus }\end{array}$ & $\begin{array}{l}1 \\
1\end{array}$ & $\begin{array}{l}0(0) \\
0(0)\end{array}$ & $\begin{array}{l}0 \\
0\end{array}$ & $\begin{array}{l}0 \\
0\end{array}$ & - & $\begin{array}{l}0 \\
0\end{array}$ & I & $\mathrm{C}$ & S & 2 & & $\mathrm{X}$ & \\
\hline $\begin{array}{l}\text { Passeriformes } \\
\text { Formicariidae (6) } \\
\text { Thamnophilus caerulescens } \\
\text { Thamnophilus torquatus } \\
\text { Dysithamnus mentalis } \\
\text { Herpsilochmus atricapillus } \\
\text { Pyriglena leucoptera } \\
\text { Conopophaga lineata }\end{array}$ & $\begin{array}{r}56 \\
17 \\
1 \\
15 \\
2 \\
4 \\
17\end{array}$ & $\begin{array}{l}14(25.0) \\
4(23.5) \\
0(0) \\
4(26.6) \\
0(0) \\
2(50.0) \\
4(23.5)\end{array}$ & $\begin{array}{r}30 \\
5 \\
0 \\
11 \\
0 \\
4 \\
10\end{array}$ & $\begin{array}{l}0.53 \\
0.29 \\
0 \\
0.73 \\
0 \\
1 \\
0.59\end{array}$ & $\begin{array}{l}\overline{2.14} \\
1.25 \\
\overline{2.75} \\
\overline{2} \\
2.5\end{array}$ & $\begin{array}{c}0-7 \\
0-2 \\
0 \\
0-7 \\
0 \\
0-3 \\
0-5\end{array}$ & $\begin{array}{l}\text { I } \\
\text { I } \\
\text { I } \\
\text { I } \\
\text { I } \\
\text { I }\end{array}$ & $\begin{array}{l}\text { A } \\
\text { A } \\
\text { A } \\
\text { A } \\
\text { F } \\
\text { A }\end{array}$ & $\begin{array}{l}\text { S } \\
\text { S } \\
\text { S } \\
\text { S } \\
\text { S } \\
\text { N }\end{array}$ & $\begin{array}{l}3 \\
1 \\
3 \\
3 \\
3 \\
3\end{array}$ & $\begin{array}{l}X \\
X \\
X \\
X \\
X\end{array}$ & $\begin{array}{l}X \\
X \\
X \\
X\end{array}$ & $\begin{array}{l}X \\
X\end{array}$ \\
\hline $\begin{array}{l}\text { Family Furnariidae (5) } \\
\text { Synallaxis ruficapilla } \\
\text { Synallaxis frontalis } \\
\text { Syndactyla rufosuperciliata } \\
\text { Automolus leucophthalmus } \\
\text { Lochmias nematura }\end{array}$ & $\begin{array}{r}19 \\
4 \\
1 \\
1 \\
11 \\
2\end{array}$ & $\begin{array}{l}2(10.5) \\
0(0) \\
0(0) \\
0(0) \\
2(18.2) \\
0(0)\end{array}$ & $\begin{array}{l}2 \\
0 \\
0 \\
0 \\
2 \\
0\end{array}$ & $\begin{array}{l}0.10 \\
0 \\
0 \\
0 \\
0.18 \\
0\end{array}$ & $\begin{array}{l}\frac{1}{-} \\
\frac{1}{-}\end{array}$ & $\begin{array}{c}0-1 \\
0 \\
0 \\
0 \\
0-1 \\
0\end{array}$ & $\begin{array}{l}\text { I } \\
\text { I } \\
\text { I } \\
\text { I } \\
\text { I }\end{array}$ & $\begin{array}{l}\mathrm{F} \\
\mathrm{F} \\
\mathrm{C} \\
\mathrm{C} \\
\mathrm{C}\end{array}$ & $\begin{array}{c}\mathrm{S} \\
\mathrm{S} \\
\mathrm{S} \\
\mathrm{S} \\
\mathrm{N}\end{array}$ & $\begin{array}{l}3 \\
3 \\
3 \\
3 \\
3\end{array}$ & $\begin{array}{l}X \\
X\end{array}$ & $\begin{array}{l}\text { X } \\
X \\
X \\
X\end{array}$ & X \\
\hline $\begin{array}{l}\text { Dendrocolaptidae (2) } \\
\text { Sittasomus griseicapillus } \\
\text { Lepidocolaptes fuscus }\end{array}$ & $\begin{array}{r}22 \\
17 \\
5\end{array}$ & $\begin{array}{c}3(13.6) \\
3(17.6) \\
0(0)\end{array}$ & $\begin{array}{l}8 \\
8 \\
0\end{array}$ & $\begin{array}{l}0.36 \\
0.47 \\
0\end{array}$ & $\begin{array}{l}2.66 \\
2.66 \\
-\end{array}$ & $\begin{array}{c}0-5 \\
0-5 \\
0\end{array}$ & $\begin{array}{l}\text { I } \\
\text { I }\end{array}$ & $\begin{array}{l}\mathrm{C} \\
\mathrm{C}\end{array}$ & $\begin{array}{l}\mathrm{S} \\
\mathrm{S}\end{array}$ & $\begin{array}{l}3 \\
3\end{array}$ & $\begin{array}{l}X \\
X\end{array}$ & $\begin{array}{l}X \\
X\end{array}$ & $\mathrm{X}$ \\
\hline $\begin{array}{l}\text { Tyrannidae (14) } \\
\text { Elaenia mesoleuca? } \\
\text { Elaenia cristata }\end{array}$ & $\begin{array}{r}88 \\
1 \\
2\end{array}$ & $\begin{array}{c}10(11.3) \\
0(0) \\
0(0)\end{array}$ & $\begin{array}{r}20 \\
0 \\
0\end{array}$ & $\begin{array}{l}0.23 \\
0 \\
0\end{array}$ & $\begin{array}{l}2.0 \\
- \\
-\end{array}$ & $\begin{array}{c}0-6 \\
0 \\
0\end{array}$ & $\begin{array}{l}F \\
F\end{array}$ & $\begin{array}{l}\text { A } \\
\text { A }\end{array}$ & $\begin{array}{l}\mathrm{N} \\
\mathrm{N}\end{array}$ & $\begin{array}{l}3 \\
1\end{array}$ & & $\begin{array}{l}X \\
X\end{array}$ & \\
\hline
\end{tabular}




\begin{tabular}{|c|c|c|c|c|c|c|c|c|c|c|c|c|c|}
\hline $\begin{array}{l}\text { Family } \\
\text { (species) }\end{array}$ & $\begin{array}{l}\text { Individuals } \\
\text { examined }\end{array}$ & Prevalence $^{a}$ & $\begin{array}{l}\text { Number } \\
\text { of ticks }\end{array}$ & $\begin{array}{l}\text { Relative } \\
\text { density } b\end{array}$ & $\begin{array}{c}\text { Mean } \\
\text { intensity }\end{array}$ & Variation $^{d}$ & Diet $^{e}$ & Nest $^{f}$ & $\begin{array}{l}\text { Mixed } \\
\text { species } \\
\text { flocks } g\end{array}$ & $\begin{array}{c}\text { Forest } \\
\text { dependence }^{h}\end{array}$ & $\begin{array}{l}\text { Forest } \\
\text { interior }\end{array}$ & $\begin{array}{l}\text { Forest } \\
\text { border }\end{array}$ & Cerrado \\
\hline Elaenia chiriquensis? & 1 & $0(0)$ & 0 & 0 & - & 0 & $\mathrm{~F}$ & A & $\mathrm{N}$ & 1 & & $\mathrm{X}$ & \\
\hline Elaenia obscura & 2 & $0(0)$ & 0 & 0 & - & 0 & $\mathrm{~F}$ & A & $\mathrm{N}$ & 3 & & & $\mathrm{X}$ \\
\hline Mionectes rufiventris & 4 & $1(25.0)$ & 1 & 0.25 & 1 & $0-1$ & $\mathrm{~F}$ & $\mathrm{~F}$ & $S$ & 3 & $\mathrm{X}$ & $\mathrm{X}$ & \\
\hline Leptopogon amaurocephalus & 9 & $0(0)$ & 0 & 0 & - & 0 & $\mathrm{I}$ & $\mathrm{F}$ & $\mathrm{S}$ & 3 & $X$ & $X$ & $X$ \\
\hline Phylloscates ventralis & 1 & $1(100)$ & 1 & 1 & 1 & 1 & I & A & $\mathrm{S}$ & 3 & $\mathrm{X}$ & & \\
\hline Hemitriccus margaritaceiventer & 1 & $0(0)$ & 0 & 0 & - & 0 & I & $\mathrm{F}$ & $\mathrm{N}$ & 2 & & $\mathrm{X}$ & \\
\hline Tolmomyias sulphurenscens & 6 & $2(33.3)$ & 4 & 0.66 & 2 & $0-6$ & $\mathrm{I}$ & $\mathrm{F}$ & $\mathrm{S}$ & 3 & $\mathrm{X}$ & $\mathrm{X}$ & \\
\hline Platyrinchus mystaceus & 29 & $3(10.3)$ & 6 & 0.21 & 2.0 & $0-2$ & $\mathrm{I}$ & A & $\mathrm{S}$ & 3 & $\mathrm{X}$ & $\mathrm{X}$ & \\
\hline Myiophobus fasciatus & 5 & $1(20.0)$ & 1 & 0.20 & 1 & $0-1$ & I & $\mathrm{F}$ & $S$ & 1 & & $\mathrm{X}$ & $\mathrm{X}$ \\
\hline Lathrotriccus euleri & 21 & $2(9.5)$ & 7 & 0.33 & 3.5 & $0-5$ & I & A & $\mathrm{S}$ & 3 & $\mathrm{X}$ & $\mathrm{X}$ & $\mathrm{X}$ \\
\hline Cnemotriccus fuscatus & 1 & $0(0)$ & 0 & 0 & - & 0 & I & A & $\mathrm{N}$ & 3 & & & $X$ \\
\hline Myiarchus ferox & 5 & $0(0)$ & 0 & 0 & - & 0 & I & $\mathrm{C}$ & $\mathrm{N}$ & 2 & & $\mathrm{X}$ & $\mathrm{X}$ \\
\hline Pipridae (3) & 43 & (9)20.9 & 46 & 1.07 & 5.1 & $0-12$ & & & & & & & \\
\hline Chiroxiphia caudata & 19 & (8) 42.1 & 43 & 2.26 & 5.4 & $0-12$ & $\mathrm{~F}$ & A & $\mathrm{N}$ & 3 & $\mathrm{X}$ & $\mathrm{X}$ & $\mathrm{X}$ \\
\hline Ilicura militaris & 23 & $0(0)$ & 0 & 0 & - & 0 & $\mathrm{~F}$ & A & $\mathrm{N}$ & 3 & $\mathrm{X}$ & $X$ & $X$ \\
\hline Schiffornis virescens & 1 & $1(100)$ & 3 & 3 & 3 & 3 & $\mathrm{I}$ & $\mathrm{C}$ & $\mathrm{N}$ & 3 & $X$ & & \\
\hline Troglodytidae (1) & 4 & $0(0)$ & 0 & 0 & - & 0 & & & & & & & \\
\hline Troglodytes aedon & 4 & $0(0)$ & 0 & 0 & - & 0 & I & A & $\mathrm{N}$ & 1 & & $\mathrm{X}$ & $\mathrm{X}$ \\
\hline Muscicapidae (5) & 35 & $6(17.1)$ & 12 & 0.34 & 2 & $0-3$ & & & & & & & \\
\hline Turdus rufiventris & 7 & $1(14.3)$ & 1 & 0.14 & 1 & $0-1$ & $\mathrm{O}$ & A & $\mathrm{N}$ & 1 & $\mathrm{X}$ & $\mathrm{X}$ & \\
\hline Turdus leucomelas & 12 & $3(25.0)$ & 7 & 0.58 & 2.33 & $0-3$ & $\mathrm{O}$ & A & $\mathrm{N}$ & 2 & $\mathrm{X}$ & $X$ & $\mathrm{X}$ \\
\hline Turdus amaurochalinus & 2 & $0(0)$ & 0 & 0 & - & 0 & $\mathrm{O}$ & A & $\mathrm{N}$ & 2 & & $\mathrm{X}$ & \\
\hline Turdus albicollis & 9 & $2(22.2)$ & 4 & 0.44 & 2 & $0-3$ & $\mathrm{O}$ & A & $\mathrm{N}$ & 3 & $\mathrm{X}$ & $\mathrm{X}$ & \\
\hline Turdus nigriceps & 5 & $0(0)$ & 0 & 0 & - & 0 & $\mathrm{O}$ & A & $\mathrm{N}$ & 3 & $\mathrm{X}$ & $\mathrm{X}$ & \\
\hline Vireonidae (3) & 6 & $1(16.6)$ & 5 & 0.83 & 5 & $0-5$ & & & & & & & \\
\hline Cyclarhis gujanensis & 1 & $1(100)$ & 5 & 5 & 5 & 5 & $\mathrm{O}$ & A & $\mathrm{S}$ & 2 & & $\mathrm{X}$ & \\
\hline Vireo olivaceus & 3 & $0(0)$ & 0 & 0 & - & 0 & $\mathrm{O}$ & A & $\mathrm{S}$ & 3 & & & $\mathrm{X}$ \\
\hline Hylophilus poicilotis & 2 & $0(0)$ & 0 & 0 & - & 0 & $\mathrm{O}$ & $\mathrm{A}$ & $S$ & 2 & & $\mathrm{X}$ & $\mathrm{X}$ \\
\hline Emberizidae (20) & 163 & $25(15.3)$ & 54 & 0.33 & 2.16 & $0-5$ & & & & & & & \\
\hline Geothlypis aequinoctialis & 4 & $0(0)$ & 0 & 0 & - & 0 & I & A & $\mathrm{N}$ & 1 & & & $\mathrm{X}$ \\
\hline Basileuterus flaveolus & 22 & $5(22.7)$ & 13 & 0.63 & 2.8 & $0-6$ & I & $\mathrm{F}$ & $S$ & 3 & $\mathrm{X}$ & $\mathrm{X}$ & $X$ \\
\hline Basileuterus hypoleucus & 29 & $1(3.44)$ & 1 & 0.03 & 1 & $0-1$ & $\mathrm{I}$ & $\mathrm{F}$ & $\mathrm{S}$ & 3 & $X$ & $X$ & $X$ \\
\hline Coereba flaveola & 7 & $0(0)$ & 0 & 0 & - & 0 & $\mathrm{~N}$ & $\mathrm{~F}$ & $\mathrm{~S}$ & 2 & & $\mathrm{X}$ & $\mathrm{X}$ \\
\hline Schistochlamys ruficapillus & 3 & $1(33.3)$ & 1 & 0.33 & 1 & $0-1$ & $\mathrm{~F}$ & A & $\mathrm{S}$ & 1 & & & $\mathrm{X}$ \\
\hline Neothraupis fasciata & 3 & $0(0)$ & 0 & 0 & - & 0 & $\mathrm{~F}$ & A & $\mathrm{S}$ & 1 & & & $\mathrm{X}$ \\
\hline Tachyphonus coronatus & 4 & $2(50.0)$ & 6 & 1.5 & 3 & $0-5$ & $\mathrm{~F}$ & A & $\mathrm{S}$ & 3 & $\mathrm{X}$ & $\mathrm{X}$ & \\
\hline
\end{tabular}




\begin{tabular}{|c|c|c|c|c|c|c|c|c|c|c|c|c|c|}
\hline $\begin{array}{l}\text { Family } \\
\text { (species) }\end{array}$ & $\begin{array}{c}\text { Individuals } \\
\text { examined }\end{array}$ & Prevalence $^{a}$ & $\begin{array}{l}\text { Number } \\
\text { of ticks }\end{array}$ & $\begin{array}{l}\text { Relative } \\
\text { density }^{b}\end{array}$ & $\begin{array}{c}\text { Mean } \\
\text { intensity }\end{array}$ & Variation $^{d}$ & Diet $^{e}$ & Nest $^{f}$ & $\begin{array}{l}\text { Mixed } \\
\text { species } \\
\text { flocks } g\end{array}$ & $\begin{array}{c}\text { Forest } \\
\text { dependence }^{h}\end{array}$ & $\begin{array}{l}\text { Forest } \\
\text { interior }\end{array}$ & $\begin{array}{l}\text { Forest } \\
\text { border }\end{array}$ & Cerrado \\
\hline Trichothraupis melanops & 29 & $8(27.5)$ & 17 & 0.58 & 2.1 & $0-3$ & $\mathrm{O}$ & A & $\mathrm{S}$ & 3 & $X$ & $\mathrm{X}$ & $X$ \\
\hline Tangara cyanocephala & 2 & $0(0)$ & 0 & 0 & - & 0 & $\mathrm{~F}$ & A & $\mathrm{N}$ & 2 & & & $\mathrm{X}$ \\
\hline Tangara cayana & 6 & $1(16.6)$ & 3 & 0.5 & 3 & $0-3$ & $\mathrm{O}$ & A & $\mathrm{S}$ & 1 & $X$ & $\mathrm{X}$ & $\mathrm{X}$ \\
\hline Dacnis cayana & 2 & $0(0)$ & 0 & 0 & - & 0 & $\mathrm{O}$ & A & $\mathrm{N}$ & 2 & & & $\mathrm{X}$ \\
\hline Zonotrichia capensis & 2 & $0(0)$ & 0 & 0 & - & 0 & $\mathrm{O}$ & A & $\mathrm{S}$ & 1 & & & $\mathrm{X}$ \\
\hline Haplospiza unicolor & 8 & $1(12.5)$ & 1 & 0.13 & 1 & 1 & $\mathrm{G}$ & A & $\mathrm{N}$ & 2 & $X$ & $X$ & $X$ \\
\hline Sicalis citrina & 1 & $0(0)$ & 0 & 0 & 一 & 0 & $\mathrm{G}$ & $\mathrm{C}$ & $\mathrm{N}$ & 1 & & & $\mathrm{X}$ \\
\hline Embernagra longicauda & 1 & $1(100)$ & 1 & 1 & 1 & 1 & G & A & $\mathrm{N}$ & 1 & & & $\mathrm{X}$ \\
\hline Volatinia jacarina & 8 & $0(0)$ & 0 & 0 & - & 0 & $\mathrm{G}$ & A & $\mathrm{N}$ & 1 & & & $\mathrm{X}$ \\
\hline Sporophila caerulescens & 6 & $0(0)$ & 0 & 0 & - & 0 & $\mathrm{G}$ & A & $\mathrm{S}$ & 1 & & $\mathrm{X}$ & $\mathrm{X}$ \\
\hline Sporophila sp. & 7 & $0(0)$ & 0 & 0 & - & 0 & $\mathrm{G}$ & A & $\mathrm{S}$ & 1 & & $X$ & $X$ \\
\hline Arremon flavirostris & 3 & $1(33.3)$ & 2 & 0.66 & 2 & $0-2$ & $\mathrm{O}$ & $\mathrm{F}$ & $\mathrm{N}$ & 3 & & $X$ & \\
\hline Saltator similis & 16 & $4(25.0)$ & 9 & 0.56 & 2.25 & $0-3$ & $\mathrm{O}$ & A & $\mathrm{N}$ & 2 & $\mathrm{X}$ & $\mathrm{X}$ & $X$ \\
\hline
\end{tabular}

$a$ : number of infested individuals (percentage of infestation); $b$ : number of ticks/number of birds examined; $c$ : number of ticks/number of birds infested; $d$ : range of number of ticks; $e$ : diet; N: nectarivore; F: frugivore; G: gramnivore; I: insectivore; O: omnivore. After Hilty and Brown (1986), and Sick (1997); $f$ :forest dependence: 1: independent;

2: semidependent; 3: dependent. After Silva (1995); $g$ : participation in mixed species flocks of birds. S: always, regular, or occasionally; N: never. After Ridgely and Tudor (1989), and Sick (1997); $h$ : nest type constructed. A: open; F: closed; C: cavity (trunk or ground). After Sick (1997).
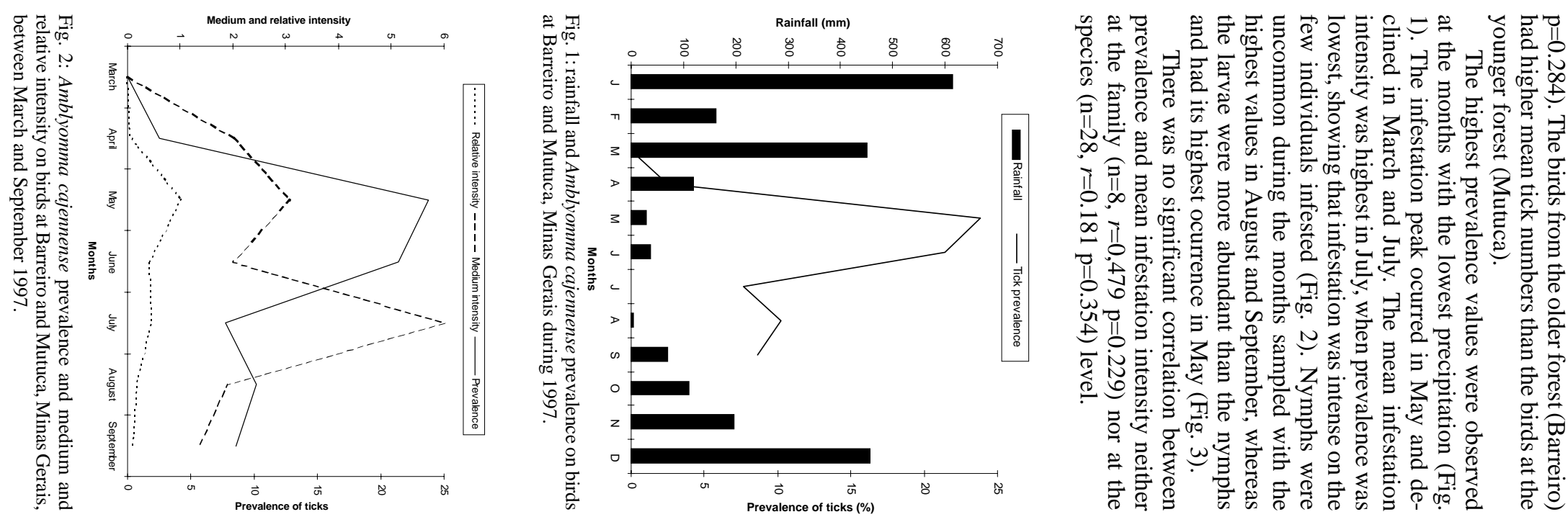
TABLE II

Prevalence, relative density and mean intensity of Amblyomma cajenense, on non-passeriformes and passeriformes birds at Barreiro and Mutuca, Minas Gerais, between March and September 1997

\begin{tabular}{lcccccc}
\hline & $\begin{array}{c}\text { Individuals } \\
\text { examined }\end{array}$ & Prevalence $^{a}$ & $\begin{array}{c}\text { Number } \\
\text { of ticks }\end{array}$ & $\begin{array}{c}\text { Relative } \\
\text { density }\end{array}$ & $\begin{array}{c}\text { Mean } \\
\text { intensity }^{c}\end{array}$ & Variation $^{d}$ \\
\hline Totals non passeriformes (8) & 31 & $0(0)$ & 0 & 0 & 0 & 0 \\
Total Passeriformes (59) & 436 & $70(16)$ & 177 & 0.40 & 2.5 & $0-12$ \\
Total (67) & 467 & $70(15)$ & 177 & 0.38 & 2.5 & $0-12$ \\
\hline
\end{tabular}

$a$ : number of infested individuals (percentage of infestation); $b$ : number of ticks/number of birds examined; $c$ : number of ticks/number of birds infested; $d$ : range of number of ticks.

TABLE III

Tick prevalence on birds of cerrado, forest border, and forest interior at Barreiro and Mutuca, Minas Gerais, between March and September 1997

\begin{tabular}{lcccc}
\hline Habitat & $\begin{array}{c}\text { Individuals } \\
\text { examined }\end{array}$ & $\begin{array}{c}\text { Tick prevalence } \\
(\%)\end{array}$ & $\begin{array}{c}\text { Chi-Square } \\
\left(\mathrm{c}_{Y}^{2}\right)\end{array}$ & Probability \\
\hline Cerrado & 90 & 10 & 2.45 & 0.1173 \\
Forest & 349 & 17 & & 0.3943 \\
Forest interior & 146 & 19.8 & 0.73 & 0.001 \\
Forest border & 203 & 15.7 & 10.86 & 0.5506 \\
Barreiro forest & 259 & 21.6 & & 0.36 \\
Mutuca forest & 90 & 5.5 & 6.8 & \\
Barreiro cerrado & 44 & 13.04 & & \\
Mutuca cerrado & 46 & & & \\
\hline
\end{tabular}

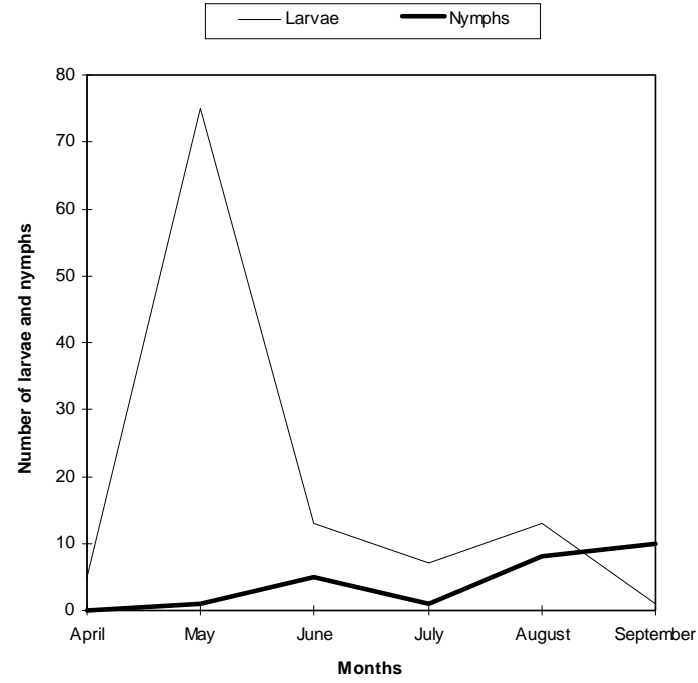

Fig. 3: number of larvae and nymphs of Amblyomma cajennense on birds at Barreiro and Mutuca, Minas Gerais, between April and September 1997.

\section{DISCUSSION}

The birds sampled seem to be secondary hosts helping in the dispersal of immature phases (larvae and nymphs) but not adults of $A$. cajennense. A. cajennense occurred on 28 of the 67 species of birds sampled, showing a lack of host specificity during its immature stages, as suggested by Hoogstraal and Aeschlimann (1982). The hosts of the adult phase of $A$. cajennense include several species of wild and domestic vertebrates (Aragão 1936).

Prevalence of Amblyomma was of $15 \%$ for all birds sampled, with $0 \%$ prevalence for nonPasseriformes and $16 \%$ for Passeriformes (Table I). These values are similar to the ones reported by Pruett-Jones and Pruett-Jones (1991) for other species of Ixodid in forest birds of New Guinea (15.4\% for all birds; $0.6 \%$ for non-Passeriformes; and $16.4 \%$ for Passeriformes). However, the total values differ from the ones reported by Marini et al. (1996) for Passeriformes from the Atlantic Forest of the State of Paraná (45.5\%) and from Marini and Couto (1997) for Passeriformes and nonPasseriformes captured at several forests of Minas Gerais $(24.3 \%)$. These data reveal a wide variation in the prevalence of ixodid ticks on birds among different geographical regions.

Our analysis also showed that there is variation in the infestation level of Amblyomma, depending on the ecology and behavior of the host. The pattern of infestation ranges from host species with high infestation levels to species which are uninfected. This is evident for Passeriformes which had high mean infestation levels and especially in 
some families (Table I). Host species with the highest prevalence also showed high mean infestation intensity at both the family and species level. This pattern was also reported by Pruett-Jones and Pruett-Jones (1991) and Marini et al. (1996). High infestation may represent low immunity in some individuals, a decrease in health state and body condition, and a decrease in the birds' movement ability which may increase the encounter rate with ticks, causing a further increase in the infestation probability (Pruett-Jones \& Pruett-Jones 1991). The low prevalence of $A$. cajennense at the sampled birds may be related to their low host specificity and to the fact that $A$. cajennense has at least three hosts during its cycle (Travassos \& Vallejo-Freire 1944).

A. cajennense have low population densities in birds, possibly due to grooming behavior which may be considered an avian defense against ectoparasites. An evidence of grooming is the fact that ticks are encountered mostly on body parts which birds' beaks cannot reach easily, such as the head and the neck.

Non-Passeriformes showed zero prevalence, probably because this group was mostly represented by hummingbirds (Trochilidae) which do not seem to host ticks, as was observed at this study and also by Marini et al. (1996) and Marini and Couto (1997). When exchanging hosts, ticks depend on the frequency of encounter with their hosts (Dobson \& May 1986) and the foraging behavior of hummingbirds may reduce the possibilities of such encounters.

According to Travassos and Vallejo-Freire (1944) and Serra Freire (1982), A. cajennense is found in its adult phase from late September to late March. Larvae, however, are more frequent during May and June, and nymphs during July and September, with some variations among localities, climate, temperature and hygrometry degree. Here we observed a pattern similar to the one described by the above authors for domestic animals (Figs 2,3).

Larvae and nymphs of $A$. cajennense can remain up to 100 days without feeding, maintaining their ability to attack hosts and to complete their life cycle (Olivieri \& Serra Freire 1984a, b, Souza \& Serra Freire 1992, Serra Freire \& Olivieri 1992). This characteristic, associated with the need for three hosts during its evolutive cycle, and their relative readiness to parasitize attacking domestic and wild animals, as well as humans, enable infected individuals to inoculate infectant material during the next phase to another animal or man (Travassos \& Vallejo-Freire 1944).

When habitats are destroyed, fragmented or modified, different potential hosts make contact with each other, allowing the exchange of their associated arthropods. If these ectoparasites are vectors, the exchange of diseases may occur. Epidemics occur easily when diseases move within new host populations which have not been exposed in their natural habitat (Gettinger \& Ernest 1995). The movement of domestic species into wild areas associated with habitat fragmentation increase this risk, which could be kept to a minimum if natural communities were kept as intact as possible, and free of domestic animals.

This makes it important to know in greater detail the role of wild species, such as birds, as dispersers of this tick. An integration between ecology and veterinary medicine will allow a better understanding of population dynamics of this tick.

\section{ACKNOWLEDGMENTS}

To the students of the Bird Ecology Laboratory (UFMG) for collaboration in the field work. To Bruce Alexander for English revision. To Paulo R Oliveira for criticisms and useful suggestions. To Cristina MN Lopes and Ramiro Botelho for help in tick identification. To Cemave (Ibama) for banding permission. To "Companhia de Saneamento de Minas Gerais" (Copasa) for permission to work at their properties.

\section{REFERENCES}

Aragão H 1936. Ixodidas brasileiros e de alguns paizes limitrophes. Mem Inst Oswaldo Cruz 31: 759-844.

Botelho JR, Linardi PM, da Encarnação CD 1989. Interrelações entre Acari Ixodidae e hospedeiros edentata da Serra da Canastra, Minas Gerais, Brasil. Mem Inst Oswaldo Cruz 84: 61-64.

Cetec 1983. Diagnóstico ambiental do estado de Minas Gerais, p. 34-42. Fundação Centro Tecnológico de Minas Gerais, Belo Horizonte.

Cetec 1996. Desenvolvimento de tecnologia para o manejo de espécies nativas e recuperação de áreas degradadas de proteção das captações da Copasa Mutuca e Barreiro. Relatório Técnico Final, Fundação Centro Tecnológico de Minas Gerais, Belo Horizonte, $55 \mathrm{pp}$.

Clifford CM, Anastos G 1960. The use of chaetotaxy in the identification of larval ticks (Acarina: Ixodidae) J Parasitol 46: 567-578.

Coutinho MTZ 1997. Guilda de Ectoparasitos de Pequenos Mamíferos para Comparações Espaciais e Temporais entre Ambientes, MSc Thesis, UFMG, Belo Horizonte, $126 \mathrm{pp}$.

Dobson AP, May RM 1986. Disease and conservation, p. 345-365. In ME Soulé, Conservation Biology. The Science of Scarcity and Diversity, Sunderland, Massachussets.

Dunning JS 1987. South American Birds. A Photographic Aid to Identification, Harrowood, Pennsylvania, 230 pp.

Famadas KM, Serra Freire NM, Lanfrendi RM 1997. Redescription of the larva of Amblyomma cajennense (Fabricius) (Acari: Ixodidae) using optical and scannig electron microscopy. Acarologia 38: 101-109.

Gettinger D, Ernest KA 1995. Small-mammal com- 
munity structure and the specificity of ectoparasite associations in central Brazil. Rev Bras Biol 55: 331-341.

Hilty SL, Brown WL 1986. A Guide to the Birds of Colombia, Princeton Univ. Press, Princeton, 836 pp.

Hoogstraal H, Aeschlimann A 1982. Tick-host specificity. Bull Societe Entomol Suisse 55: 5-32.

Linardi PM, Teixeira VC, Botelho JR, Riveiro LS 1987. Ectoparasitos de roedores em ambientes silvestres do município de Juiz de Fora, Minas Gerais. Mem Inst Oswaldo Cruz 82: 137-139.

Lopes C, Leite R, Labruna M, Oliveira P, Borges L, Rodriquez Z, Carvalho H, Freitas C, Júnior C 1998. Host specifity of Amblyomma cajennense (Fabricius, 1787) (Acari Ixodidae) with comments on the dropoff rhythm. Mem Inst Oswaldo Cruz 93: 347-351.

Margolis L, Esch GW, Holmes JC, Kuris AM, Schad GA 1982. The use of ecological terms in parasitology (report of an ad hoc committee of the American Society of Parasitologists). J Parasitol 68: 131-133.

Marini MÂ, Couto D 1997. Correlações ecológicas entre ectoparasitos e aves de floresta de Minas Gerais, p. 210-218. In LL Leite, CH Saito (eds), Contribuições ao Conhecimento Ecológico do Cerrado, Depto de Ecologia, Universidade de Brasília, Brasília.

Marini MÂ, Reinert BL, Bornschein MR, Pinto JC, Pichorim MA 1996. Ecological correlates of ectoparasitism on Atlantic Forest birds, Brazil. Ararajuba 4: 93-103.

Olivieri JA, Serra Freire NM 1984a. Estádio larval do ciclo biológico de Amblyomma cajennense. Arq Univ Fed Rur RJ 7: 139-148.

Olivieri JA, Serra Freire NM 1984b. Estádio ninfal do ciclo biológico de Amblyomma cajennense. Arq Univ Fed Rur RJ 7: 149-156.

Pruett-Jones S, Pruett-Jones MA 1991. Analysis and ecological correlates of tick burdens in a New Guinea avifauna, p.154-176. In JE Loye \& M Zuk (eds), Bird-parasite Interactions: Ecology, Evolution and Behaviour, Oxford Univ. Press, Oxford.

Ridgely RS, Tudor G 1989 a. The Birds of South America, Vol. I, Univ. Texas Press, Austin, 616 pp.

Ridgely RS, Tudor G 1989 b. The Birds of South America, Vol. II, Univ. Texas Press, Austin, 814 pp.

Rohr CJ 1909. Estudos sobre Ixodidae do Brasil. Rio de Janeiro. Mem Inst Oswaldo Cruz 1: 110-117.

Serra Freire NM 1982. Epidemiologia de Amblyomma cajennense: ocorrência estacional e comportamento dos estádios não parasitários em pastagens do estado do Rio de Janeiro. Arq Univ Fed Rur RJ 5: 187-193.

Serra Freire NM, Cunha DW 1987. Amblyomma cajennense: comportamento de ninfas e adultos como parasitos de bovinos. Rev Bras Med Vet 9: 100-103.

Serra Freire NM, Olivieri JA 1992. Estádio adulto do ciclo de Amblyomma cajennense. Arq Fac Vet UFRGS 20: 224-234.

Sick H 1997. Ornitologia Brasileira, Nova Fronteira, Rio de Janeiro, 858 pp.

Silva JMC 1995. Birds of the cerrado region, South America. Steenstrupia 21: 69-92.

Smith MW 1974. Some aspects of the ecology and lifecycle of Amblyomma cajennense (Fabricius, 1787 ) in Trinidad and their influence on tick control measures. Ann Trop Med Parasitol 69: 121-129.

Souza AP, Serra Freire NM 1992. Variação sazonal dos estádios adultos de Amblyomma cajennense e Anocentor nitens, como parasitas de cavalos, no município de Itaguaí, RJ, Brasil. Rev Brasil Parasitol Vet 1: 31-34.

Travassos J, Vallejo-Freire A 1944. Criação artificial de Amblyomma cajennense para o preparo da vacina contra a febre maculosa. Mem Inst Butantan 18: 145235.

Walker A 1994. Arthropods of Humans and Domestic Animals, Chapman and Hall, London, 213 pp. 